\title{
ETIOLOGY AND FREQUENCY OF RESPIRATORY DISTRESS IN NEONATES: A CROSS SECTIONAL STUDY CONDUCTED IN TERTIARY CARE HOSPITAL, LAHORE.
}

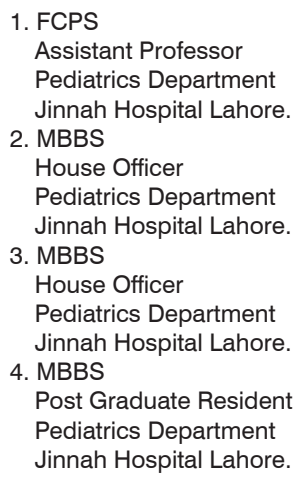

Correspondence Address: Dr. Naila Jabbar Pediatrics Department Jinnah Hospital Lahore. dr.nailajabbar@gmail.com

Article received on: 22/10/2018

Accepted for publication: 06/03/2019

\begin{abstract}
Mirza Liaqat Ali', Naila Jabbar' ${ }^{2}$, Abdul Hannan ${ }^{3}$, Azher ${ }^{4}$
ABSTRACT: Respiratory distress in neonate defines as when rate of respiration is greater than sixty in one minute, nasal flaring, grunting and intercostal / sub coastal recession is present. Various causes are reported of respiratory distress in new born. Objectives: To determine frequency of respiratory distress in new born and to Find frequency of various etiologies of respiratory distress in full term newborn admitted up to the age of 24 hours of birth in Study Design: Cross sectional studies. Setting: Neonatal unit of Jinnah Hospital Lahore. Period: From February 10, 2016 till August 10, 2016. Material and Method: SPSS v-20 was used for analysis of data like name, age, sex, gestational age, maternal record of pregnancy and fetus for etiologies of respiratory distress. Results: There were 100(66.7\%) male and 50(33.3\%) females in this study. The mean gestational age of these newborn was $39.51 \pm 1.51$ weeks. Respiratory distress was seen in $16(10.7 \%)$ of the cases. Transient tachypnea seen in $4(25 \%)$ of the cases, Meconium aspiration syndrome was diagnosed in 2(12.5\%), Pneumothorax in $3(18.8 \%)$, Congenital pneumonia in 2 (12.5\%), Sepsis in 6(37.5\%) and Birth asphyxia 2 (12.5\%) of early neonatal age were the common observed causes. Conclusion: Respiratory distress was $1 / 10$ of the cases. The commonest etiologies were Sepsis preceding to Respiratory distress syndrome, MAS (Meconium Aspiration Syndrome), TTN (Transient Tacyhpnea of Newborn), Pneumothorax, Congenital pneumonia, Birth asphyxia.
\end{abstract}

Key words: Causes, Complication, Distress, Etiology, Infants, Lungs, Respiratory, Risk.

Article Citation: Ali ML, Jabbar N, Hannan A, Azher. Etiology and frequency of respiratory distress in neonates: A cross sectional study conducted in tertiary care hospital, Lahore. Professional Med J 2019; 26(11):1815-1819.

DOI: 10.29309/TPMJ/2019.26.11.245

\section{INTRODUCTION}

Respiratory distress is considered as a serious emergency in newborns. It demands urgent attention and timely intervention. Recognition of these signs and treatment is important because the patient can rapidly deteriorate to respiratory failure. ${ }^{1}$ Respiratory distress in neonate defines as when rate of respiration is greater than sixty in one minute, nasal flaring, grunting and intercostal/sub coastal recession is present. About $20 \%$ of NICU admissions are due to respiratory distress. ${ }^{2}$ It may be due to infections, anatomic obstruction, URTI and LRTI and poor respiratory effort secondary to CNS dysfunction. Respiratory distress may also be due to transient tachypnea or serious conditions like meconium aspiration and surfactant deficiency. ${ }^{3}$ In a study, transient tachypnea of the newborns (50.8\%) showed the highest incidence, followed by pneumonia (20.3\%), extra pulmonary infection $(11.9 \%)$, respiratory distress syndrome $(6.8 \%)$, air leak (5.1\%), meconium aspiration syndrome (3.9\%). ${ }^{4}$ In a study carried out on 553 neonates, about 76 (13.7\%) developed respiratory distress. In new born, transient tachypnea was found in $46 \%$ followed by hyaline membrane disease in $31.5 \%$, birth asphyxia in $25 \%$, pneumonia and sepsis in $25 \%$, MAS $7.8 \%$, pneumothorax $2.6 \%$, congenital heart disease (PDA) $1.3 \%$ and laryngomalacia $1.3 \% .^{5}$ A prospective study was carried out in North India on 1400 neonates, out of which $116(8.2 \%)$ developed respiratory distress 67 neonates were term and 49 preterm. In term infants, major cause was of pneumonia and Bronchopneumonia (51.4\%). Other etiologies include TTN 22(19\%). MAS 14(12.1\%) and was frequently observed in full term newborns than preterm newborns. Hyaline membrane 
disease 10(86\%). Two cases were diagnosed as Pneumothorax. ${ }^{6}$ The rationale of this study was to collect data from local population for determining frequency of respiratory distress. This study also determined etiology of respiratory distress as early recognition of underlying cause can help in earlier treatment which can improve prognosis of many preventable causes.

\section{MATERIAL AND METHODS}

\section{Study Design}

Cross sectional study.

\section{Study Setting}

Department of Pediatrics, Jinnah Hospital, Lahore.

\section{Duration of Study}

Half year after permission of ethical review board of Allama lqbal Medical College. From Feb 10, 2016 till Aug 10, 2016

\section{Sample Size}

Sample size of 150 cases, calculated from winpepi version 11.15, with 95\% Confidence level having $8 \%$ verge of error and estimated percentage of RDS is $6.8 \%{ }^{4}$ In neonates presenting within 24 hours of life with respiratory distress.

\section{Sample Technique}

Non-probability consecutive sampling.

\section{Sample Selection}

Neonates presenting within 24 hours of birth to neonatal unit of Jinnah Hospital Lahore. Neonates with congenital abnormalities like cleft palate and cleft lip (on clinical examination) and trachoesophageal fistula.

\section{Data Collection Procedure}

A total of 150 neonates, who fulfill the inclusion and exclusion criteria, were recruited in the study from Emergency Department of Pediatrics Medicine, Jinnah Hospital Lahore. Informed consent was obtained from parents. Demographic details like name, age, gestational age at birth and gender was obtained. Antenatal record of mother and neonate was assessed thoroughly for causes of respiratory distress. All information was recorded on study Performa. Through clinical examination and chest $\mathrm{X}$-rays that was done and respiratory distress and its causes was labeled as per operational definition.

\section{Statistical Analysis}

The data was entered and analysis was done through SPSS v-20. Standard deviation and mean were measured for all variables like gestational age and age. Percentage and frequency was measured for respiratory distress and causes like infection, birth asphyxia, MAS, TTN, sepsis, congenital pneumonia, RDS, pneumothorax. Figures were stratified for gender (male / female) and gestational age at birth (37-40, 40-42weeks). Stratified groups were compared for causes of respiratory distress using "chi-square" test. Significant $p$-value was taken as less than and equal to 0.05

\section{RESULTS}

The mean age of newborn was $10.43 \pm 7.38$ hours with minimum and maximum age of 1 and 23 hours (Table-I). The mean gestational age of these now born was $39.51 \pm 1.51$ with minimum and maximum gestational age of 37 and 42 weeks (Table-I). There were 100(66.7\%) male and $50(33.3 \%)$ females in this study. In this study respiratory distress was seen in 16(10.7\%) of the cases. We found, transient tachypnea, the most communal cause of respiratory distress of Newborn 4(25\%) as, MAS (Meconium Aspiration Syndrome) was diagnosed in $2(12.5 \%)$ of the cases, RDS(Respiratory Distress Syndrome) in $3(18.8 \%)$ of the infants, Congenital pneumonia in $2(12.5 \%)$, Pneumothorax in 3(18.8\%), Sepsis in $6(37.5 \%)$ and Birth asphyxia was seen 2 (12.5\%) of the new born (Table-III). When data was stratified for gender and gestational age we found no significant association of respiratory distress with gender ( $p$-value >0.05) and there was significant association of respiratory distress with gestational age, p-value $<0.05$. Most of the causes of respiratory distress were not necessarily associated with gestational age and gender only Congenital pneumonia was profoundly associated with gestational age ( $p$-value < 0.05) while Respiratory distress and 
Sepsis was associated with gender ( $p$-value $<0.05$ ) (Table-III) Frequency distribution of causes of the respiratory distress $[n=16]$. (TableII) Comparison of congenial pneumonia with gestational age $[n=16]$.

\begin{tabular}{|l|c|c|}
\hline \multicolumn{1}{|c|}{ Statistics } & $\begin{array}{c}\text { Gestational Age } \\
\text { (weeks) }\end{array}$ & Age (hours) \\
\hline Mean & 39.51 & 10.4367 \\
\hline Std. Deviation & 1.514 & 7.37793 \\
\hline Range & 5 & 22.00 \\
\hline Minimum & 37 & 1.00 \\
\hline Maximum & 42 & 23.00 \\
\hline
\end{tabular}

Table-I. There were 100(66.7\%) male and 50(33.3\%) females in this study.

\begin{tabular}{|l|c|c|c|c|}
\hline \multicolumn{2}{|c|}{} & \multicolumn{2}{|c|}{$\begin{array}{c}\text { Congenital } \\
\text { Pneumonia }\end{array}$} & \multirow{2}{*}{ P-Value } \\
\cline { 2 - 4 } & & Yes & No & \\
\hline \multirow{2}{*}{$\begin{array}{l}\text { Gestational } \\
\text { Age (week) }\end{array}$} & $37-39$ & 2 & 2 & \\
\cline { 2 - 4 } & $40-42$ & $100 \%$ & $14.3 \%$ & \multirow{2}{*}{0.009} \\
\cline { 2 - 4 } & & 0 & 12 & \\
\hline \multirow{2}{*}{ Total } & $.0 \%$ & $85.7 \%$ & \\
\hline & & 2 & 14 & 16 \\
\hline
\end{tabular}

Table-II. Comparison of congenial pneumonia with gestational age $[n=16]$

\begin{tabular}{|l|c|c|}
\hline \multicolumn{1}{|c|}{ Cause } & Present & Absent \\
\hline Transient tachypnea of new born & $4(25 \%)$ & $12(75 \%)$ \\
\hline Meconium aspiration syndrome & $2(12.5 \%)$ & $14(87.5 \%$ \\
\hline Respiratory distress syndrome & $3(18.8 \%)$ & $13(81.2 \%)$ \\
\hline Congenital pneumonia & $2(12.5 \%)$ & $14(87.5 \%)$ \\
\hline Pneumothorax & $3(18.8 \%)$ & $13(81.2 \%)$ \\
\hline Sepsis & $6(37.5 \%)$ & $10(62.5 \%)$ \\
\hline Birth asphyxia & $2(12.5 \%)$ & $14(87.5 \%)$ \\
\hline
\end{tabular}

Table-III. Frequency distribution of causes of the respiratory distress $[n=16]$

\section{DISCUSSION}

Respiratory distress is a very commonly encountered problem in newborn. ${ }^{7}$ In NICU of Youngman University Hospital had done a study on neonate admitted soon after birth with tachypnea, there were male predominance at $2.65: 1 .{ }^{4} \mathrm{We}$ in current found that the mean age was $10.43 \pm 7.38$ hours and there were $100(66.7 \%)$ male and $59(33.3 \%)$ female patients.
We also found male are increase in number and ratio is $2: 1$. These results are identical to above mentioned findings. Respiratory distress occurred in up to $7 \%$ of full term neonates which were admitted during early days of life in different nurseries of worldwide hospitals. ${ }^{8}$ In current study the prevalence of respiratory distress was seen in $10.7 \%$ newborn that is considerably higher prevalence when compared with above cited study. The elective C-section deliveries has also elevated the occurrence of TTN. Moreover, the risk of respiratory distress is decreases by increasing the gestational age. The risks are three times more at post-term gestation than at term gestation. TTN, RDS, pneumonia, MAS, pneumothorax and persistent pulmonary hypertension are the most observed causes in term neonates. ${ }^{8}$ In this study transient tachypnea of new born was seen $4(25 \%)$ of the cases, Meconium aspiration syndrome was diagnosed in $2(12.5 \%)$ of the cases, Respiratory distress syndrome in $3(18.8 \%)$ of the infants, Congenital pneumonia in 2 (12.5\%), Pneumothorax in $3(18.8 \%)$, Sepsis in $6(37.5 \%)$ and Birth asphyxia was seen $2(12.5 \%)$ of the new born was common causes of respiratory distress. A recent study deduced that the usual cases of Respiratory distress seen in the study were birth asphyxia 254 (24.66\%), Hyaline membrane disease 262 (25.44\%), Neonatal Sepsis/Pneumonia 242 (23.50\%), MAS 73 (7.09\%). Uncommon causes were Congenital heart Disease 44 (4.27\%), Tracheoesophageal fistula 54 (5.24\%), TTN 43 $(4.17 \%)$ and others $58(5.63 \%) .^{9}$ They reported that incidence of respiratory distress is more in male compare to female. ${ }^{9}$ These findings are comparable to our findings. Another research concluded that RDS occurred in 10.5 percent and TTN is $6.4 \%$ in which $8.5 \%$ were simply given oxygen with nasal prongs, $6.6 \%$ required intubation and ventilator support and $2.8 \%$ required high-frequency oscillatory ventilation (HFOV). ${ }^{10}$ Misra PK et al found the causes of respiratory distress in neonates were pneumonia $(32.2 \%)$, transient tachypnea of new born (18.7\%), CNS causes (asphyxia, hemorrhage) (17.7\%), Meconium aspiration syndrome (15\%), IRDS (12.5\%), Miscellaneous (3.2\%). ${ }^{11}$ Kumar et al also observed that Transient tachypnea of new 
born (48.4\%), HMD (Hyaline Membrane Disease) (27.7\%), meconium aspiration syndrome $(10.3 \%)$, pneumonia $(2.3 \%)$ and miscellaneous $(12.3 \%)$ were communal reasons of respiratory distress in neonate. ${ }^{12}$ Rubalteli FF, Dani C, Reali MF et al found the reason of respiratory distress in neonates were HMD (46\%), pneumonia/sepsis $(5 \%)$, transient tachypnea of new born (37\%), congenital cardiac malformation (2\%), meconium aspiration syndrome (2\%), multiple congenital anomalies (1.4\%), perinatal asphyxia (1.1\%), spontaneous pneumothorax (1.2\%) and others $(4.3 \%) .{ }^{13}$ These findings are comparable to our findings as in our series we found pneumothorax was the most common cause in our study. In another study carried out at a tertiary care hospital following etiological spectrum was observed, transient tachypnea of the newborns $(50.8 \%)$ showed the highest incidence, followed by pneumonia $(20.3 \%)$, extra pulmonary infection (11.9\%), respiratory distress syndrome $(6.8 \%)$, air leak (5.1\%), meconium aspiration syndrome (3.9\%). ${ }^{4}$ In a study carried out in India it was noted that 553 neonates got admission in nursery throughout the study period, out of which 76 (13.7\%) neonates developed respiratory distress, Transient tachypnea of new born was found in $46 \%$ followed by hyaline membrane disease in $31.5 \%$, birth asphyxia in $25 \%$, pneumonia and sepsis in $25 \%$, MAS $7.8 \%$, pneumothorax $2.6 \%$, congenital heart disease (PDA) $1.3 \%$ and laryngomalacia $1.3 \% .{ }^{5} \mathrm{~A} 13$ month period prospective study in India was carried out among 1400 neonates to determine cause of respiratory distress. 116 (8.2\%) developed respiratory distress in which 67 were term and 49 were preterm. Majority of the cases $(51.4 \%)$ were cause due to bronchopneumonia. Transient tachypnea was observed in 22 cases (19\%). Meconium aspiration was held responsible in 14 cases $(12.1 \%)$ and was common in term infants than preterm ones. Hyaline membrane disease was cause in 10 cases (86\%). Pneumothorax was observed in 2 cases. ${ }^{6}$ The difference of these statistics may be due to the sample size, gestational age and locality the patients. More studies are requested to confirm the statistics of current study by taking larger sample size.

Copyright@ 06 March, 2019.

\section{REFERENCES}

1. Gortner L, Tutdibi EJZfGuN. Respiratorische Erkrankungen bei Früh-und Neugeborenen-Aktuelle Diagnostik und Therapie. 2011; 215(04):145-51.

2. Qian L-I, Liu C-q, Guo Y-x, Jiang Y-j, Ni L-m, Xia S-w, et al. Current status of neonatal acute respiratory disorders: a one-year prospective survey from a Chinese neonatal network. 2010; 123(20):2769-75.

3. Ma X-I, Xu X-f, Chen C, Yan C-y, Liu Y-m, Liu L, et al. Epidemiology of respiratory distress and the illness severity in late preterm or term infants: A prospective multi-center study. 2010; 123(20):2776-80.

4. Seo JH, Lee KH, Lee ESJYUJoM. Clinical courses and diagnoses of neonates who are transferred due to mild respiratory distress soon after birth in a university hospital. 2014; 31(2):89-93.

5. Santosh S, Kumar K, Adarsha EJIJNMR. A clinical study of respiratory distress in newborn and its outcome. 2013; 1(1):2-4.

6. Thomas S, Verma IC, Singh M, Menon PJTIJoP. Spectrum of respiratory distress syndrome in the newborn in North India: A prospective study. 1981; 48(1):61-5.

7. Paul VJlp. Neonatal morbidity and mortality: Report of the national neonatal and perinatal database. 1999; 36(2):167.

8. Edwards MO, Kotecha SJ, Kotecha SJPrr. Respiratory distress of the term newborn infant. 2013; 14(1):2937.

9. Bajad M, Goyal S, Jain BJIJoCP. Clinical profile of neonates with respiratory distress. 2016; 3(3):100913.

10. Hibbard JU, Wilkins I, Sun L, Gregory K, Haberman $\mathrm{S}$, Hoffman $\mathrm{M}$, et al. Respiratory morbidity in late preterm births. 2010; 304(4):419.

11. Mathur N, Garg K, Kumar SJlp. Respiratory distress in neonates with special reference to pneumonia. 2002; 39(6):529-38.

12. Kumar A, Bhat BVJTIJoP. Epidemiology of respiratory distress of newborns. 1996; 63(1):93-8.

13. Rubaltelli F, Dani C, Reali M, Bertini G, Wiechmann L, Tangucci $\mathrm{M}$, et al. Acute neonatal respiratory distress in Italy: a one-year prospective study. 1998; 87 (12):12618. 


\begin{tabular}{|c|c|c|c|}
\hline \multicolumn{4}{|c|}{ AUTHORSHIP AND CONTRIBUTION DECLARATION } \\
\hline Sr. \# & Author(s) Full Name & Contribution to the paper & Author(s) Signature \\
\hline 1 & Mirza Liaqat Ali & \multirow{4}{*}{$\begin{array}{l}\text { All author have equal } \\
\text { contributions. }\end{array}$} & \\
\hline 2 & Naila Jabbar & & Taila tolnear \\
\hline 3 & Abdul Hannan & & Abdul Hennaur \\
\hline 4 & Azher & & $b^{3}=$ \\
\hline
\end{tabular}

